\title{
PP242 suppresses bladder cancer cell proliferation and migration through deactivating the mammalian target of rapamycin complex 2/AKT1 signaling pathway
}

\author{
ZHE ZHANG $^{1}$, GUOJUN ZHANG ${ }^{2}$, CHUIZE KONG $^{1}$ and DAXIN GONG ${ }^{1}$ \\ ${ }^{1}$ Department of Urology, The First Hospital of China Medical University, Shenyang, Liaoning 110001; \\ ${ }^{2}$ Department of Hematology, Shengjing Hospital of China Medical University, Shenyang, Liaoning 110022, P.R. China
}

Received January 1, 2015; Accepted October 6, 2015

DOI: $10.3892 / \mathrm{mmr} .2015 .4528$

\begin{abstract}
While most cancer types are resistant to mammalian target of rapamycin complex 1 (mTORC1) inhibitor rapamycin, recent studies have identified mTORC2 as an important prospective therapeutic target for cancer. The present study assessed the effects of mTORC2 inhibitor PP242 on the proliferation and migration of bladder cancer cells by using Cell Counting Kit-8, 5-ethynyl-2'-deoxyuridine incorporation, wound healing and Transwell assays. Furthermore, the phosphorylation status of downstream signaling proteins of mTORC1 and mTORC2 was assessed using western blot analysis. The results demonstrated that PP242 concentration-dependently inhibited the proliferation of bladder cancer cells. Simultaneously, the migration ability of bladder cancer cells was suppressed by PP242. In addition, PP242 markedly restrained the phosphorylation of AKT1 and mTORC2, while the phosphorylation status of S6K1 and mTORC1 was not affected. These results suggested that PP242 exerts potent inhibitory effects on bladder cancer cells by modulating the activity of the mTORC2/AKT1 pathway.
\end{abstract}

\section{Introduction}

As the most common malignant tumor type of the urinary tract, bladder cancer remains one of the major causes of cancer-associated mortality worldwide (1). Due to its high recurrence rate and requirement of costly lifelong follow-ups, the development of novel and effective therapies for bladder cancer is necessary, for which the elucidation of the underlying mechanisms of oncogenic urothelial cell transformation is vital.

Correspondence to: Professor Daxin Gong, Department of Urology, The First Hospital of China Medical University, 155 Nanjing North Street, Heping, Shenyang, Liaoning 110001, P.R. China

E-mail: lauren1944@sina.com

Key words: bladder cancer, mammalian target of rapamycin, PP242, cell proliferation, AKT1, S6K1
Mammalian target of rapamycin (mTOR) has a central role in regulating cellular catabolism and anabolism and is important in tumorigenesis and cancer progression (2). mTOR kinase exists as mTOR complex (mTORC)1 and 2, two distinctive cellular protein complexes, which each have a unique molecular composition, substrates and mechanisms of activation (3). mTORC1 regulates autonomous cell growth in dependent on the availability of growth factors and nutrients, whereas mTORC2 regulates cell survival and proliferation (4). Upon interaction of raptor with mTOR, mTORC1 is formed, which represents the sensitive target of rapamycin that phosphorylates downstream targets of eukaryotic initiation factor 4E binding protein-1 (4E-BP1) and S6 kinase 1 (S6K1). The mTORC1 signaling pathway has a central role in regulating cell functions, including proliferation, growth, survival and mobility (5-7). Aberrant expression of mTORC1 has been observed in numerous types of cancer, including colorectal cancer (8), hepatocellular carcinoma (9), renal cell carcinoma (10), breast cancer (11), acute myeloid leukemia (12) and non-small cell lung cancer (13). Although mTOR research largely focuses on mTORC1, mTORC2 is emerging as a crucial signaling complex in numerous cancer types. It has been reported that mTORC2 directly phosphorylates AKT on serine (ser)473, which leads to the activation of this anti-apoptotic kinase and ultimately results in increased cell survival, proliferation and migration (14).

The first-generation mTOR inhibitor rapamycin partially suppresses mTORC1 activity and reduces the proliferation of cancer cells (15); however, rapamycin is not sufficiently potent for the effective treatment of cancer. For this reason, efforts have been made to develop selective small-molecule competitors of adenosine triphosphate (ATP) as mTOR inhibitors with the ability to completely block mTORC1 effectors in addition to mTORC2 substrates (16). The present study examined the anti-proliferative and anti-migratory effects of PP242, an ATP-competitive inhibitor that binds the mTOR catalytic site, in bladder cancer cells. It was indicated that PP242 reduced the viability and proliferation of bladder cancer cells and inhibited their migratory potential. In addition, PP242 reduced the mTORC2/AKT1 activity, however did not effect mTORC1/S6K1 activity in bladder cancer cells. 


\section{Materials and methods}

Cell lines and reagents. The SV-HUC-1, RT4, BIU-87, 5637 and T24 bladder cancer cell lines were purchased from the Cell Bank of the Chinese Academy of Sciences (Shanghai, China). All cell lines were maintained in a humidified atmosphere with $5 \% \mathrm{CO}_{2}$ at $37^{\circ} \mathrm{C}$. The cell lines were grown in RPMI-1640 (GE Healthcare Life Sciences, Logan, UT, USA) media containing fetal bovine serum (GE Healthcare Life Sciences) according to the manufacturer's instructions.

PP242 was purchased from Selleck Chemicals Company (Houston, TX, USA). For in-vitro experiments, PP242 was dissolved in dimethyl sulfoxide (DMSO; Sigma-Aldrich, St. Louis, MO, USA).

The rabbit polyclonal mTOR (ab2732), rabbit monoclonal raptor (ab40768) and rabbit polyclonal rictor (ab70374) antibodies were purchased from Abcam (Cambridge, MA, USA). Rabbit polyclonal phosphorylated (p)-mTOR (Ser2448) (\#2971), rabbit polyclonal p-mTOR (Ser2481) (\#2974), rabbit monoclonal p-S6K1 (Thr389) (\#9234), rabbit monoclonal p-AKT1 (Ser473) (\#4058) and rabbit monoclonal GAPDH (\#2118) antibodies were obtained from Cell Signaling Technology, Inc. (Danvers, MA, USA). Mouse monoclonal AKT1 (sc-5298) and rabbit polyclonal S6K1 (sc-230) antibodies were from Santa Cruz Biotechnology, Inc. (Dallas, TX, USA).

Cell growth and proliferation assays. The Cell Counting Kit-8 assay (CCK-8, KeyGen Biotech Co., Nanjing, China) was used to assess effects of PP242 on bladder cancer cell growth according to the manufacturer's instructions. Cells were incubated with PP242 at 100, 200, 500 or 1,000 nM or with DMSO $(<0.5 \%)$ alone for $48 \mathrm{~h}$. The absorbance was measured at a wavelength of $450 \mathrm{~nm}$ using a plate reader (model 680; Bio-Rad Laboratories, Hercules, CA, USA). In addition, the 5-ethynyl-2'-deoxyuridine (EdU) incorporation assay with the Cell-Light ${ }^{\mathrm{TM}}$ EdU DNA Cell Proliferation kit (Ruibo Biotech, Guangzhou, China) was used to assess the effects of PP242 at $500 \mathrm{~nm}$ for $48 \mathrm{~h}$ on the proliferation of bladder cancer cells according to the manufacturer's instructions. All cells were treated with $50 \mu \mathrm{mol} / \mathrm{l}$ of EdU for $24 \mathrm{~h}$ at $37^{\circ} \mathrm{C}$. Following being fixed with $4 \%$ paraformaldehyde for $15 \mathrm{~min}$, the cells were treated with $0.5 \%$ Triton X-100 (Sigma-Aldrich, St. Louis, MO, USA) for $20 \mathrm{~min}$ and rinsed with PBS three times. Thereafter, the cells were exposed to $100 \mu \mathrm{l}$ of $1 \mathrm{X}$ Apollo ${ }^{\circledR}$ reaction cocktail for $30 \mathrm{~min}$ and incubated with $5 \mu \mathrm{g} / \mathrm{ml}$ of Hoechst 33342 (Ruibo Biotech, Guangzhou, China) to stain the cell nuclei for $30 \mathrm{~min}$. EdU incorporation of the cells was observed under an Olympus IX-71 inverted fluorescence microscope (Olympus, Tokyo, Japan) and images were captured.

Wound healing migration assay. Cells were incubated in the presence or absence of PP242 (500 nM) or with DMSO alone for $48 \mathrm{~h}$. Following serum starvation of confluent bladder cancer cells for $2 \mathrm{~h}$ in 24-well plates, the media were replaced with RPMI-1640 medium without supplementation and line-shaped wounds were generated across the cell monolayers using a sterile pipette tip. At 0 and $24 \mathrm{~h}$, images of the wounded cell layers were captured using an Olympus
IX-71 inverted fluorescence microscope (Olympus). ImageJ software (National Institutes of Health, Bethesda, MD, USA) was used to quantify the amount of wound closure, which resembled the migratory potential of the cells.

Transwell assay. The lower chambers of a Transwell plate (with $8.0 \mu \mathrm{m}$-pore polycarbonate membranes; Corning Life Science, Corning, NY, USA) were filled with $0.6 \mathrm{ml}$ RPMI-1640 medium containing 5\% FBS. Cells were incubated in the presence or absence of PP242 (500 nM) or with DMSO alone for $48 \mathrm{~h}$. Cells were re-suspended in RPMI-1640 containing 1\% FBS and $200 \mu \mathrm{l}$ cell suspension $\left(1 \times 10^{6}\right.$ cells $\left./ 100 \mathrm{ml}\right)$ were added to the upper chamber. After $24 \mathrm{~h}$ of incubation at $37^{\circ} \mathrm{C}$ with $5 \% \mathrm{CO}_{2}$, cells which had not migrated were removed using cotton swabs, whereas migrated cells were fixed in $4 \%$ paraformaldehyde (Shanghai Haochen Biological Technology Co., Ltd., Shanghai, China) for $10 \mathrm{~min}$ at room temperature, stained with $0.1 \%$ crystal violet (Santa Cruz Biotechnology, Inc.) and counted under an Olympus IX-71 inverted fluorescence microscope. Experiments were performed in triplicate.

Western blot analysis. The cells were lysed in radioimmunoprecipitation assay buffer (Beyotime Institute of Biotechnology, Shanghai, China) mixed with protease inhibitor (phenylmethanesulfonyl fluoride; Beyotime Institute of Biotechnology) and equal quantities of protein were subjected to western blot analysis as described previously (17). Protein content was determined by the Bicinchoninic Acid kit (Pierce Biotechnology, Inc., Rockford, IL, USA). The primary antibodies were incubated on the membranes overnight at $4^{\circ} \mathrm{C}$ in Tris-buffered saline with Tween 20. The expression of mTOR, p-mTOR, AKT1, p-AKT1, S6K1, p-S6K1 was detected using specific antibodies and GAPDH was used as the loading control.

Statistical analysis. Values are expressed as the mean \pm standard deviation of three independent experiments. SPSS 17.0 (SPSS Inc., Chicago, IL, USA) was used for statistical analysis. The paired t-test was used to analyze the results of the western blot analysis. Groups with or without treatment were compared by using one-way analysis of variance. $\mathrm{P}<0.05$ was considered to indicate a statistically significant difference between values.

\section{Results}

PP242 dose-dependently inhibits the proliferation of bladder cancer cells. To determine the potency of PP242 against bladder cancer, its ability to inhibit the proliferation of four bladder cancer cell lines, RT4, BIU-87, 5637 and T24, was assessed using the CCK- 8 and EdU incorporation assays. As shown in Fig. 1A, treatment with 100-1,000 nM PP242 for $48 \mathrm{~h}$ reduced the viability of the four bladder cancer cell lines in a dose-dependent manner, with 5637 and T24 showing higher sensitivity to PP242 than RT4 and BIU-87 $(\mathrm{P}<0.05)$. Therefore, the 200-nM concentration of PP242, which was close to the $\mathrm{IC}_{50}$ on the 5637 and $\mathrm{T} 24$ cell lines in the presence of serum, was used in the subsequent experiments, which were performed on 5637 and T24 cells. 

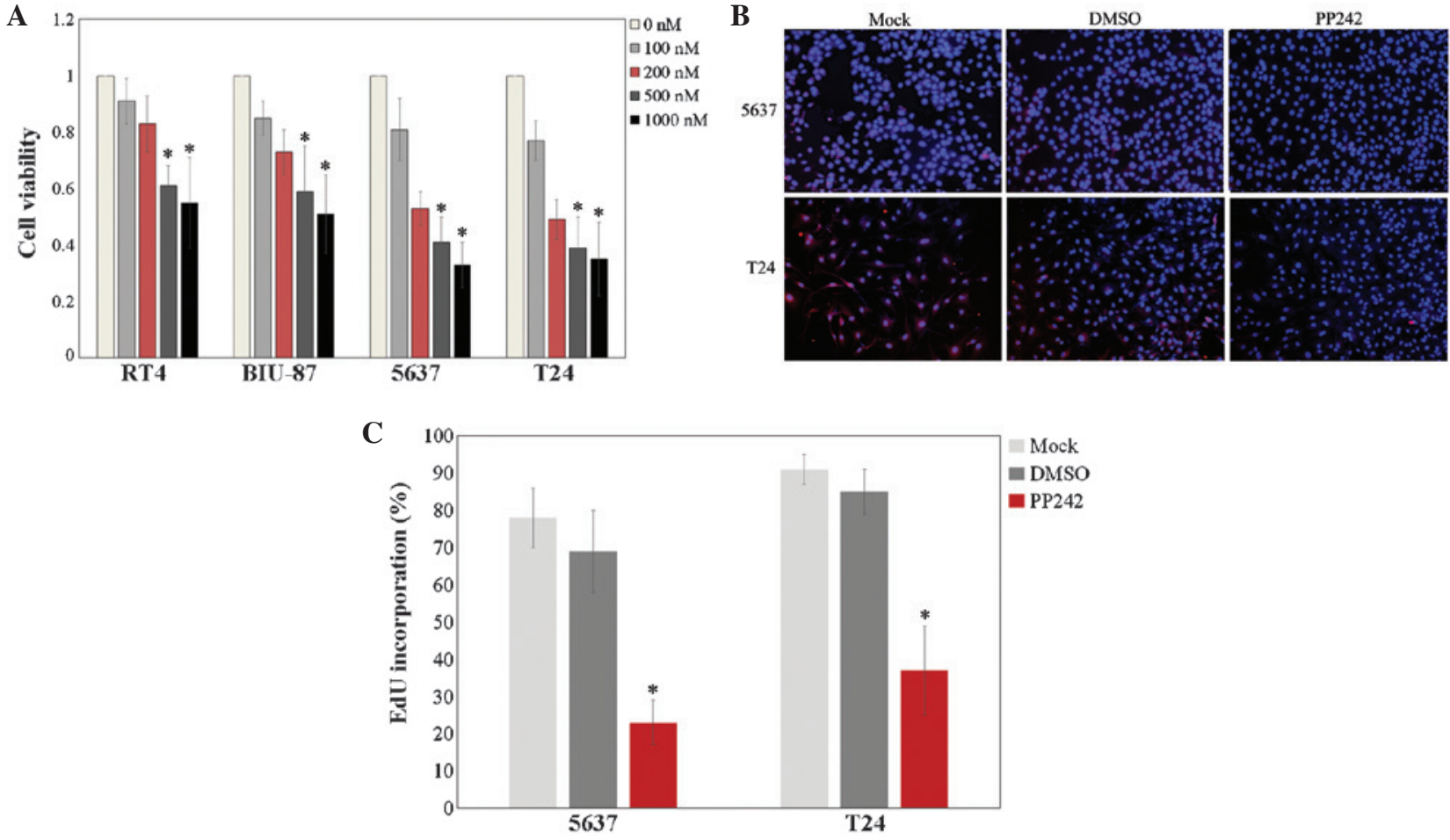

Figure 1. PP242 inhibits growth and proliferation of four bladder cancer cell lines. (A) A cell counting kit-8 assay was used to examine the viability of RT4 BIU-87, 5637 and T24 cells following treatment with PP242 (0, 100, 200, 500 or 1,000 nM for 48 h). "P<0.05 vs. 0, 100 and $200 \mathrm{nM}$. (B) Proliferation of 5637 and T24 cells was assessed by EdU incorporation with or without the treatment of PP242 ( $200 \mathrm{nM}, 48 \mathrm{~h}$ ). EdU (red) was used to stain the proliferative cells, and Hoechst 33324 (blue) was used to stain the nuclei. Images are representative of three individual experiments (magnification, x100). (C) Quantification of EdU incorporation in 5637 and T24 cells with or without the treatment of PP242 (200 nM, 48 h). "P $<0.05$ vs. Mock and DMSO. Values are expressed as the mean \pm standard deviation of three experiments. EdU, 5-ethynyl-2'-deoxyuridine; DMSO, dimethyl sulfoxide; Mock, untreated control.
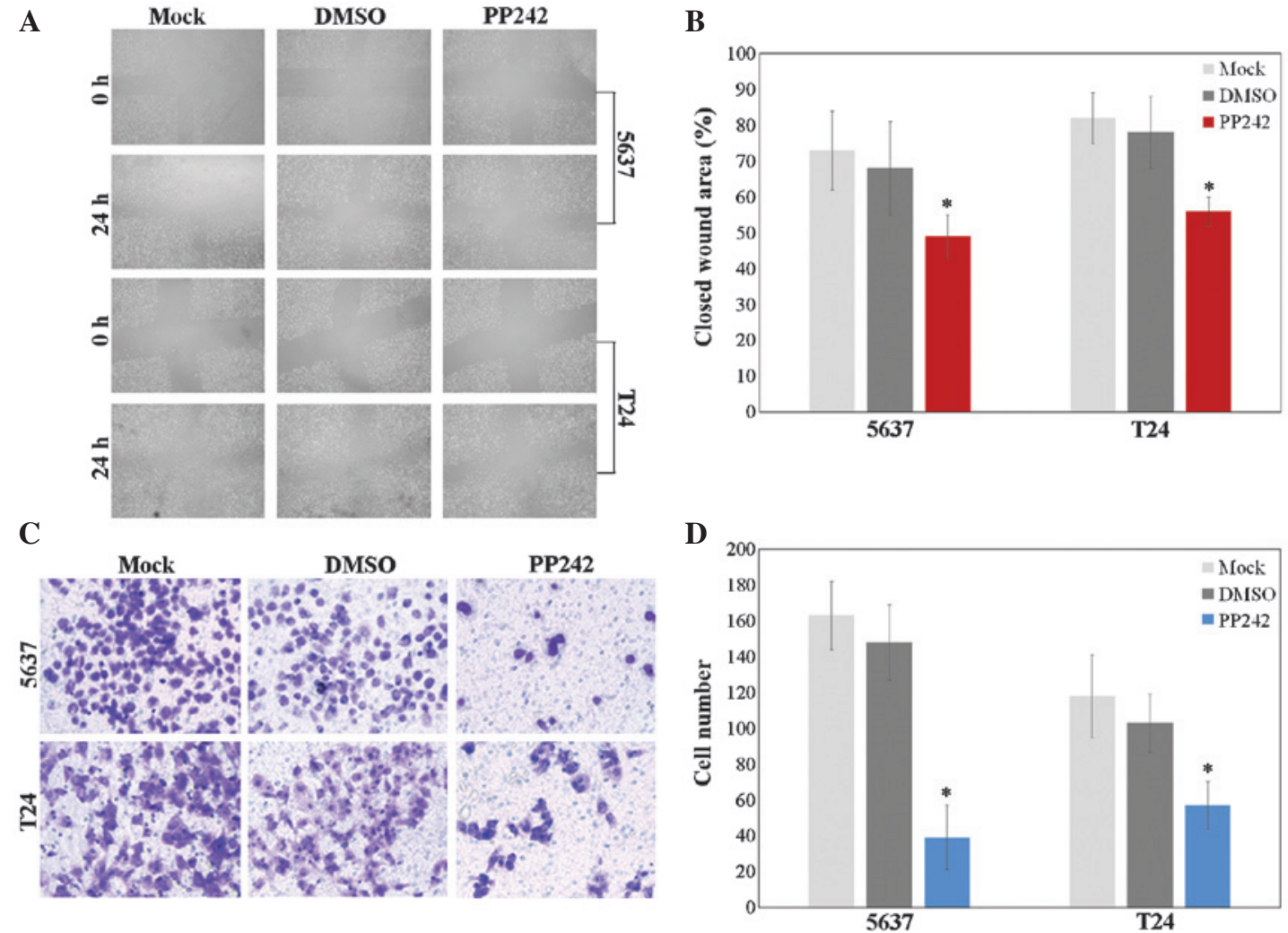

Figure 2. PP242 restrains the migration ability of bladder cancer cell lines. (A) A wound-healing assay was used to examine the migration of 5637 and T24 cells following treatment with vehicle or PP242 (200 nM, 48 h). (B) Wound closure was correlated to cell migration ability. (C) Images of 5637 and T24 cells which transgressed through Transwell membranes following treatment with vehicle or PP242 (200 nM, $48 \mathrm{~h}$ ). (D) Quantified numbers of migrated cells in C. Images are representative of three individual experiments and values are expressed as the mean \pm standard deviation; ${ }^{*} \mathrm{P}<0.05$ vs. Mock and DMSO. DMSO, dimethyl sulfoxide; Mock, untreated control. 
A

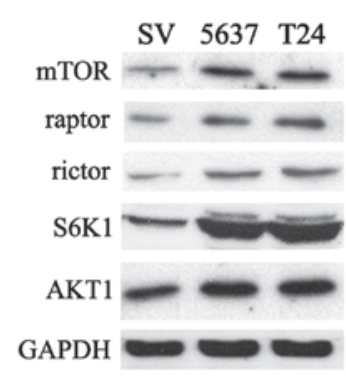

C

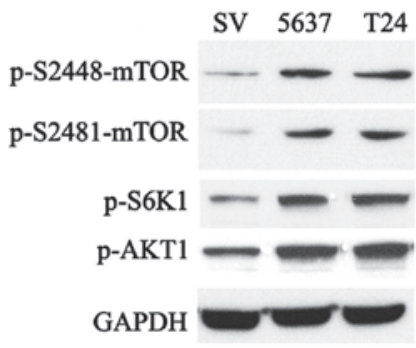

B

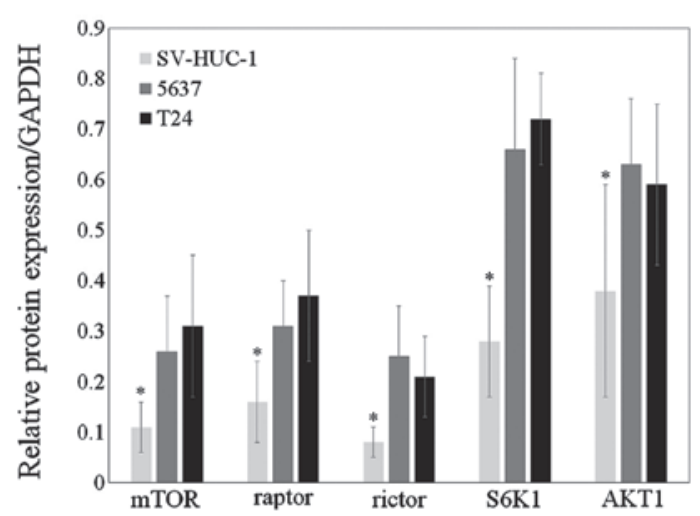

D

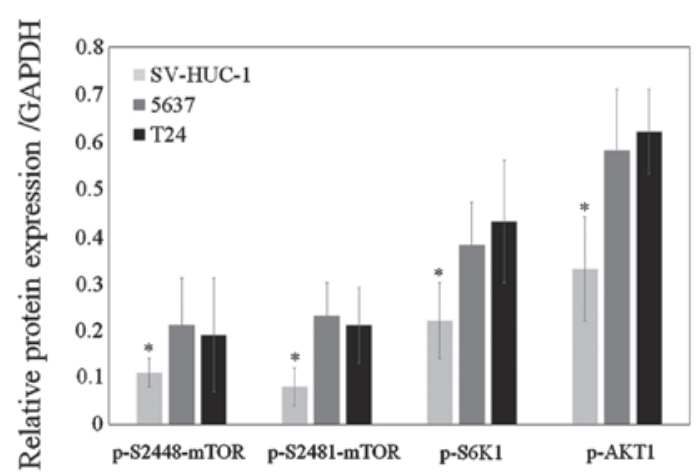

Figure 3. Expression and activation of signaling proteins of the mTOR pathway in 5637 and T24 cells. (A) Western blot analysis of mTOR, raptor, rictor, AKT1 and S6K1 in SV-HUC-1, 5637 and T24 cells. (B) Densitometric quantification of band intensities in A relative to GAPDH. (C) Western blot analysis of phosphorylated mTOR, AKT1 and S6K1 in SV-HUC-1, 5637 and T24 cells. (D) Densitometric quantification of band intensities in C relative to GAPDH. mTOR, mammalian target of rapamycin. Blots are representative of three individual experiments and values are expressed as the mean \pm standard deviation; ${ }^{*} \mathrm{P}<0.05$ vs. 5637 and T24. p, phosphorylated; mTOR, mammalian target of rapamycin; S6K1, S6 kinase 1; SV, normal bladder epithelial cell line SV-HUC-1.

A

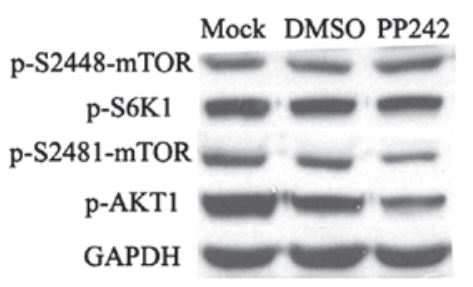

B

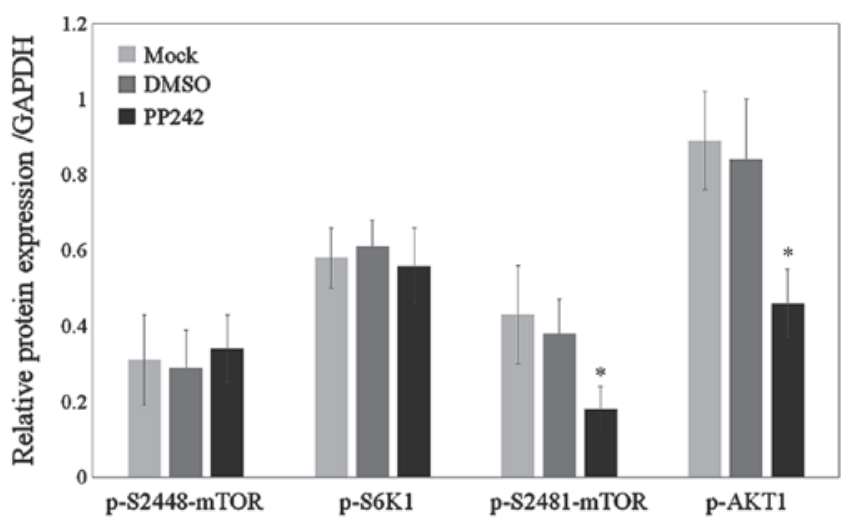

Figure 4. PP242 downregulates mTORC2/AKT1 activity but not mTORC1/S6K1 activity in bladder cancer cells. (A) Representative western blot of phosphorylated mTOR, AKT1 and S6K1 in 5637 cells treated with vehicle or PP242. (B) Densitometric quantification of band intensities in A relative to GAPDH. Values are expressed as the mean \pm standard deviation of three individual experiments; " $\mathrm{P}<0.05$ vs. Mock and DMSO. DMSO, dimethylsulfoxide, p, phosphorylated; mTOR, mammalian target of rapamycin; S6K1, S6 kinase 1; Mock, untreated control.

The EdU incorporation assay showed that following $48 \mathrm{~h}$ of incubation, $200 \mathrm{nM}$ PP2 42 decreased the mean percentage of proliferating cells by $39 \%$ for 5637 cells and by $43 \%$ for T24 cells (Fig. 1B and C; $\mathrm{P}<0.05$ ). All of these results indicated that PP242 exerts anti-proliferative effects on bladder cancer cells.

PP242 inhibits the migration of bladder cancer cells. A wound-healing assay was performed to assess the effect of
PP242 (200 nM) on the migratory ability of bladder cancer cells following $48 \mathrm{~h}$ of incubation. A wound healing assay demonstrated that wound healing of 5637 and T24 cells treated with PP242 was reduced compared to that of the negative control or DMSO-treated cells (Fig. 2A and B; $\mathrm{P}<0.05$ ).

Next, a Transwell assay was used to test the chemotactic motility of cells treated with $200 \mathrm{nM}$ PP2 42 for $48 \mathrm{~h}$. The results showed a significant decrease in the number of 5637 and T24 cells on the lower side of the Transwell membrane 
following treatment with PP242 compared with that in the control or DMSO-treated group (Fig. 2C and D; P<0.05).

Hyper-activation of the mTORC1 and mTORC2 in bladder cancer cells. As PP242 is an ATP-competitive inhibitor which binds the catalytic site of mTOR, the present study assessed whether it curtails mTORC1/2 activity. The expression and activation of signaling proteins of the mTOR pathway in 5637 and T24 cells was therefore assessed using western blot analysis. The protein expression of mTOR, raptor, rictor, AKT1 and S6K1 was shown to be elevated in 5637 and T24 cells compared with that in SV-HUC-1, an immortalized bladder epithelial cell line (Fig. 3A and B; $\mathrm{P}<0.05$ ). Selective phosphorylation of S6K1 on threonine 389 is indicative of TORC1 activity, while AKT1 phosphorylation at the ser473 residue is characteristic for TORC2 activity $(5,7)$. Furthermore, phosphorylation of mTOR on ser2481 leads to the generation of the active form of the TORC 2 complex, whereas phosphorylation of mTOR on ser2448 generates the activated form of the TORC1 complex (18). In the present study, western blot analysis showed that the phosphorylation of mTOR at ser2481 and ser2448 as well as the TORC-specific phosphorylation of S6K1 and AKT1 were all increased in 5637 and T24 cells compared with those in the SV-HUC-1 normal bladder epithelial cell line (Fig. 3C and D; $\mathrm{P}<0.05$ ). These results therefore indicated that $\mathrm{mTORC} 1$ as well as mTORC2 were activated in bladder cancer cells.

PP242 downregulates mTORC2/AKT1 activity but not mTORC1/S6K1 activity in bladder cancer cells. In order to elucidate the potential molecular mechanism via which PP242 inhibits cell proliferation, the effects of PP242 on the activation of mTORC1 and mTORC2 activation in 5637 cells were assessed. Western blot analysis revealed that following treatment with PP2 42 for $48 \mathrm{~h}$, the amount of mTOR phosphorylated on ser2448 and S6K1 phosphorylated on threonine 389 was not obviously different from those in the control and DMSO-treated groups. However, the amount of mTOR phosphorylated on ser2481 and AKT1 phosphorylated on ser473 was significantly decreased following treatment with PP242 (Fig. 4A and B; P<0.05). These results demonstrated that PP242 suppressed cell proliferation, at least in part, by deactivating the mTORC2/AKT1 signaling pathway.

\section{Discussion}

As the fourth most common type of cancer, bladder cancer occurs three times more frequently in men than in women in the USA. An estimated 74,690 of novel cases of urinary bladder cancer were diagnosed in the USA in 2014 (19), of which $70 \%$ were of the non-muscle-invasive type, which has a tendency to recur in the same or another region of the bladder at either the same stage as that of the initial tumor or a more advanced stage (20). The ability to migrate is important for the formation of metastasis/recurrence of non-muscle invasive bladder cancer. Therefore, the treatment of bladder cancer is mostly directed at reducing recurrences and preventing progression. Although combined chemotherapy initially produces high response rates, most patients experience recurrences, and the majority of them succumb to the disease shortly thereafter (21). Aberrant expression of mTORC1/mTORC2 has been observed in numerous types of cancer (8-13) and their inhibitor, PP242, has been demonstrated to reduce the activity of mTORC2/AKT1, thus decreasing cell survival, proliferation and migration (14). Accordingly, the development of more efficient anti-cancer agents is urgently required to improve the outcome of bladder cancer. The present study showed that the mTORC1/2 inhibitor PP242 inhibited the growth, proliferation and migration of bladder cancer cells.

Previous studies have shown that PP242 can restrain the growth of gastric cancer cell lines (22) and clearly demonstrated its enhanced efficacy compared with that of rapamycin in endometrial tumor models (23). PP242 is a selective ATP-competitive mTOR inhibitor and inhibits mTORC1 as well as mTORC2 simultaneously, thereby preventing the feedback activation loop of AKT and therefore exerting a greater anti-tumor activity than rapamycin and its analogues (24). The present study demonstrated that PP242 is a potent inhibitor of mTORC2 in bladder cancer cells and suppresses their proliferation in a dose-dependent manner. These inhibitory effects of PP242 were demonstrated to be mediated by its targeting of TORC2 but not TORC1. Mechanistic studies indicated that PP242 selectively inhibited the phosphorylation of AKT1 at Ser473, the downstream substrate of mTORC2, while it did not affect the phosphorylation of S6K1, the downstream substrate of mTORC1 (25). The results of the current study demonstrate that the role of PP242 in bladder cancer cells is more important for mTORC2, however further research is required to confirm the exact mechanism. Furthermore, numerous previous studies demonstrated that PP242 inhibited cell proliferation and induced apoptosis by its targeting of TORC2 (26-30). In order to confirm that PP242 targets mTORC2, the present study also examined the phosphorylation of mTOR. After treatment with PP242, phosphorylation of mTOR on ser2448, which is an activation site of mTORC1 (31) was not affected, while phosphorylation of mTOR on ser2481, an activation site of mTORC2 (32), was obviously decreased. These results indicated that mTORC2 was a target of PP242 in bladder cancer cells.

Furthermore, the present study identified that the migratory ability of bladder cancer cells was decreased by PP242 treatment, suggesting that the mTORC2 pathway also participates in the process of tumor progression. Recent studies showed that PP242 inhibited cancer-cell migration by blocking the mTOR pathway in certain tumor types $(22,33,34)$. Further study is required to determine the complex regulatory role of TORC2 signaling in the migration of bladder cancer cells.

In conclusion, the present study supported the concept that PP242 exerts its anti-tumor effects through inhibiting cell proliferation and migration by suppressing mTORC2, but not the mTORC1, in bladder cancer cells. Indeed, pre-clinical studies have indicated that PP242 is a potent anti-cancer drug.

\section{Acknowledgements}

The present study was supported by the Liaoning Province Science and Technology Plan Project (no. 2012225016), the Liaoning Provincial Natural Science Foundation (no. 2013021066) and the Shenyang City Project of Key Laboratory (no. F13-293-1-00). 


\section{References}

1. Zuo W, Wang ZZ and Xue J: Artesunate induces apoptosis of bladder cancer cells by miR-16 regulation of COX-2 expression. Int J Mol Sci 15: 14298-14312, 2014.

2. Chang H, Peng X, Bai Q, Zhou Y, Yu X, Zhang Q, Zhu J and Mi M: Ampelopsin suppresses breast carcinogenesis by inhibiting the mTOR signaling pathway. Carcinogenesis 35: 1847-1854, 2014.

3. Rivas DA, Yaspelkis BB III, Hawley JA and Lessard SJ: Lipid-induced mTOR activation in rat skeletal muscle reversed by exercise and 5'-aminoimidazole-4-carboxamide-1-beta -D-ribofuranoside. J Endocrinol 202: 441-451, 2009.

4. Wahane SD, Hellbach N, Prentzell MT, Weise SC, Vezzali R, Kreutz C, Timmer J, Krieglstein K, Thedieck K and Vogel T: PI3K-p110-alpha-subtype signalling mediates survival, proliferation and neurogenesis of cortical progenitor cells via activation of mTORC2. J Neurochem 130: 255-267, 2014.

5. Zhang Z, Zhang G, Xu X, Su W and Yu B: MTOR-rictor is the Ser473 kinase for AKT1 in mouse one-cell stage embryos. Mol Cell Biochem 361: 249-257, 2012.

6. Jash S, Dhar G, Ghosh U and Adhya S: Role of the mTORC1 complex in satellite cell activation by RNA-induced mitochondrial restoration: Dual control of cyclin D1 through microRNAs. Mol Cell Biol 34: 3594-3606, 2014.

7. Goodman CA: The role of mTORC1 in regulating protein synthesis and skeletal muscle mass in response to various mechanical stimuli. Rev Physiol Biochem Pharmacol 166: 43-95, 2014.

8. Faller WJ, Jackson TJ, Knight JR, Ridgway RA, Jamieson T, Karim SA, Jones C, Radulescu S, Huels DJ, Myant KB, et al MTORC1-mediated translational elongation limits intestinal tumour initiation and growth. Nature 517: 497-500, 2015.

9. Kaibori M, Shikata N, Sakaguchi T, Ishizaki M, Matsui K, Iida H, Tanaka Y, Miki H, Nakatake R, Okumura T, et al: Influence of rictor and raptor expression of mtor signaling on long-term outcomes of patients with hepatocellular carcinoma. Dig Dis Sci 60: 919-928, 2015.

10. Randall JM, Millard F and Kurzrock R: Molecular aberrations, targeted therapy and renal cell carcinoma: Current state-of-the-art. Cancer Metastasis Rev 33: 1109-1124, 2014.

11. Treilleux I, Arnedos M, Cropet C, Wang Q, Ferrero JM, Abadie-Lacourtoisie S, Levy C, Legouffe E, Lortholary A, Pujade-Lauraine E, et al: Translational studies within the TAMRAD randomized GINECO trial: Evidence for mTORC1 activation marker as a predictive factor for everolimus efficacy in advanced breast cancer. Ann Oncol 26: 120-125, 2015.

12. Sandhöfer N, Metzeler KH, Rothenberg M, Herold T, Tiedt S, Groiß V, Carlet M, Walter G, Hinrichsen T, Wachter O, et al: Dual PI3 K/mTOR inhibition shows antileukemic activity in MLL rearranged acute myeloid leukemia. Leukemia 29: 828-838, 2015.

13. Yue H, Li W, Liu P, Gao J, Miao J and Zhao J: Inhibition of autophagy promoted sphingosylphosphorylcholine induced cell death in non-small cell lung cancer cells. Biochem Biophys Res Commun 453: 502-507, 2014.

14. Joha S, Nugues AL, Hétuin D, Berthon C, Dezitter X, Dauphin V, Mahon FX, Roche-Lestienne C, Preudhomme C, Quesnel B and Idziorek T: GILZ inhibits the mTORC2/AKT pathway in BCR-ABL(+) cells. Oncogene 31: 1419-1430, 2012.

15. Zheng X, Liang Y, He Q, Yao R, Bao W, Bao L, Wang Y and Wang Z: Current models of mammalian target of rapamycin complex 1 (mTORC1) activation by growth factors and amino acids. Int J Mol Sci 15: 20753-20769, 2014.

16. Huang Y, Xi Q, Chen Y, Wang J, Peng P, Xia S and Yu S: A dual mTORC1 and mTORC2 inhibitor shows antitumor activity in esophageal squamous cell carcinoma cells and sensitizes them to cisplatin. Anticancer Drugs 24: 889-898, 2013.

17. Zhang Z, Zhang G and Kong C: High expression of Cdc25B and low expression of 14-3-3 $\sigma$ is associated with the development and poor prognosis in urothelial carcinoma of bladder. Tumour Biol 35: 2503-2512, 2014.
18. Fan W, Cheng K, Qin X, Narsinh KH, Wang S, Hu S, Wang Y, Chen Y, Wu JC, Xiong L and Cao F: MTORC1 and mTORC2 play different roles in the functional survival of transplanted adipose-derived stromal cells in hind limb ischemic mice via regulating inflammation in vivo. Stem Cells 31: 203-214, 2013.

19. Baack Kukreja JE, Scosynev E, Brasachio RA, Toy EP, Messing EM and Wu G: Bladder cancer incidence and mortality in patients treated with radiation for uterine cancer. BJU Int 114: 844-851, 2014.

20. Kamat AM, Vlahou A, Taylor JA, Hudson ML, Pesch B, Ingersoll MA, Todenhöfer T, van Rhijn B, Kassouf W, Barton Grossman $\mathrm{H}$, et al: Considerations on the use of urine markers in the management of patients with high-grade non-muscle-invasive bladder cancer. Urol Oncol 32: 1069-1077, 2014.

21. Petrelli F, Coinu A, Cabiddu M, Ghilardi M, Vavassori I and Barni S: Correlation of pathologic complete response with survival after neoadjuvant chemotherapy in bladder cancer treated with cystectomy: A meta-analysis. Eur Urol 65: 350-357, 2014.

22. Xing X, Zhang L, Wen X, Wang X, Cheng X, Du H, Hu Y, Li L, Dong B, Li Z and Ji J: PP242 suppresses cell proliferation, metastasis and angiogenesis of gastric cancer through inhibition of the PI3K/AKT/mTOR pathway. Anticancer Drugs 25: 1129-1140, 2014.

23. Korets SB, Musa F, Curtin J, Blank SV and Schneider RJ: Dual mTORC1/2 inhibition in a preclinical xenograft tumor model of endometrial cancer. Gynecol Oncol 132: 468-473, 2014.

24. Zhou HY and Huang SL: Current development of the second generation of mTOR inhibitors as anticancer agents. Chin J Cancer 31: 8-18, 2012

25. Chen L, Xu B, Liu L, Liu C, Luo Y, Chen X, Barzegar M, ChungJ and Huang S: Both mTORC1 and mTORC2 are involved in the regulation of cell adhesion. Oncotarget 30: 7136-7150, 2015.

26. Ravichandran K, Zafar I, Ozkok A and Edelstein CL: An mTOR kinase inhibitor slows disease progression in a rat model of polycystic kidney disease. Nephrol Dial Transplant 30: 45-53, 2015.

27. Qin Y, Zhao X and Fang Y: PP242 synergizes with suberoylanilide hydroxamic acid to inhibit growth of ovarian cancer cells. Int J Gynecol Cancer 24: 1373-1380, 2014.

28. Goncharov DA, Kudryashova TV, Ziai H, Ihida-Stansbury K, DeLisser H, Krymskaya VP, Tuder RM, Kawut SM and Goncharova EA: Mammalian target of rapamycin complex 2 (mTORC2) coordinates pulmonary artery smooth muscle cell metabolism, proliferation and survival in pulmonary arterial hypertension. Circulation 129: 864-874, 2014.

29. Becker MN, Wu KJ, Marlow LA, Kreinest PA, Vonroemeling CA, Copland JA and Williams CR: The combination of an mTORc1/TORc2 inhibitor with lapatinib is synergistic in bladder cancer in vitro. Urol Oncol 32: 317-326, 2014.

30. Bogani C, Bartalucci N, Martinelli S, Tozzi L, Guglielmelli P, Bosi A and Vannucchi AM; Associazione Italiana per la Ricerca sul Cancro AGIMM Gruppo Italiano Malattie Mieloproliferative: MTOR inhibitors alone and in combination with JAK2 inhibitors effectively inhibit cells of myeloproliferative neoplasms. PLoS One 8: e54826, 2013.

31. Müller J, Ehlers A, Burkhardt L, Sirma H, Steuber T, Graefen M, Sauter G, Minner S, Simon R, Schlomm T and Michl U: Loss of pSer2448-mTOR expression is linked to adverse prognosis and tumor progression in ERG-fusion-positive cancers. Int J Cancer 132: 1333-1340, 2013.

32. Vazquez-Martin A, Oliveras-Ferraros C, Bernadó L, López-Bonet E and Menendez JA: The serine 2481-autophosphorylated form of mammalian Target of Rapamycin (mTOR) is localized to midzone and midbody in dividing cancer cells. Biochem Biophys Res Commun 380: 638-643, 2009.

33. Wang X, Lai P, Zhang Z, Huang M, Wang L, Yin M, Jin D, Zhou R and Bai X: Targeted inhibition of mTORC2 prevents osteosarcoma cell migration and promotes apoptosis. Oncol Rep 32: 382-388, 2014.

34. Li H, Lin J, Wang X, Yao G, Wang L, Zheng H, Yang C, Jia C, Liu A and Bai X: Targeting of mTORC2 prevents cell migration and promotes apoptosis in breast cancer. Breast Cancer Res Treat 134: 1057-1066, 2012. 